Instructions for authors, subscriptions and further details:

http://rise.hipatiapress.com

\title{
Overcoming Disadvantage in Education
}

Sandra Romero Martín ${ }^{1}$

1) University of Zaragoza, Spain

Date of publication: October 25th, 2018

Edition period: October 2018 - February 2019

To cite this article: Romero Martín, S. (2018). Overcoming Disadvantage in Education [Review of the Book]. International Journal of Sociology of Education, 7(3), 300-302. doi: 10.17583/rise.2018.3807

To link this article: $\mathrm{http}: / / \mathrm{dx}$.doi.org/10.17583/rise.2018.3807

\section{PLEASE SCROLL DOWN FOR ARTICLE}

The terms and conditions of use are related to the Open Journal System and to Creative Commons Attribution License (CC-BY) 



\section{Reviews}

Gorard, S. \& See, B.H. (2013). Overcoming Disadvantage in Education. New York: Routledge

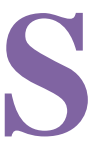

tephen Gorard, profesor de educación y políticas públicas y miembro del Wolfson Research Institute, y la investigadora Beng Huat See, ambos de la Universidad de Durham, presentan una obra que, desde una visión crítica y utilizando el modelo de causalidad en las relaciones sociales, trata de mostrar las limitaciones de los estudios académicos actuales, buscando promover intervenciones educativas más equitativas orientadas a los colectivos en situaciones desfavorecidas.

Tomando como punto de partida la premisa de que la mejora de los resultados educativos del alumnado, sobre todo del que se encuentran en entornos más desfavorecidos, es uno de los retos fundamentales de responsables políticos y académicos, señalan que muchas de las investigaciones educativas realizadas carecen del rigor y calidad suficiente para basar las decisiones políticas en base a éstas. Defienden la necesidad de trascender los análisis parciales y situar el foco en las bases de las desigualdades educativas, señalando además algunas de las áreas de estudio que consideran de vital importancia para el diseño de intervenciones más efectivas. Para ello, los autores plantean tres elementos. En primer lugar, en lo referente a las preguntas de investigación, critican su planteamiento erróneo, así como la incorrecta elección de fuentes, en ocasiones sesgadas. Por otra parte, a partir de evidencias primarias y secundarias, señalan la carencia de modelos de análisis pertinentes y efectivos sobre las causas de las desventajas educativas. Por último, tratan de identificar las principales variables indicativas de dichas desventajas, distinguiendo entre causas fijas (como es el caso de la historia familiar) y causas modificables (como la influencia de profesorado y compañeros o la segregación por características económicas entre las escuelas), siendo estas últimas las que pueden verse influenciadas por las intervenciones educativas. 
En cuanto a la estructura de la obra, en el primer capítulo se utiliza el modelo de causalidad en las ciencias sociales para revelar las relaciones causa-efecto entre los determinantes generadores de desventajas, mostrando su carácter medible y secuencial y señalando cuáles de ellos son susceptibles de ser modificados en pro de la reducción de dichas desventajas. Posteriormente, en el segundo y tercer capítulo, los autores valoran el nivel de calidad de los datos existentes, haciendo referencia a la falta de rigor y sistematización en las definiciones y formas de medida del concepto de desventaja educativa, y señalando su uso arbitrario en muchas investigaciones, lo que genera errores y sesgos en la información obtenida. En los capítulos cuatro a once, se recogen algunas de las investigaciones más relevantes sobre desventajas educativas para, a partir de éstas, señalar los desafíos metodológicos más importantes. Ponen su atención en elementos clave, como la estratificación entre escuelas y en el interior de estas, el impacto del personal docente y de la comunidad o el papel de los padres y madres, destacando especialmente el cuarto capítulo, centrado en la atención a la diversidad en el ámbito educativo. Por su parte, el último capítulo enfatiza los enfoques que, según los autores, pueden resultar más significativos para generar conocimiento científico basado en evidencias.

Gorard y See explican que, a partir de su modelo, se descubre la relación entre diversidad escolar y mayor rendimiento del alumnado desfavorecido, lo que apunta a una relación causal entre segregación y vulnerabilidad. Bajo este argumento, consideran que la diversidad debe ser promovida desde las propias escuelas públicas. Además, se muestran de nuevo críticos frente a anteriores investigaciones, que han negado las relaciones causales entre variables como la participación de padres y madres, o el nivel de calidad de centros y equipos docentes, y la desventaja educativa. Para los autores, los centros educativos deben cumplir el imperativo de representar la diversidad de las comunidades amplias en las que se encuentran, compensando de este modo la segregación socioeconómica que se observa dentro de los barrios que componen dichas comunidades, y generando un escenario que represente para el alumnado "el tipo de sociedad que deseamos tener, en lugar de reflejar las desigualdades de la sociedad que realmente tenemos". 
Se muestran partidarios por tanto de un mayor control en la composición escolar para garantizar la diversidad, señalándola como la tarea educativa de mayor importancia a desarrollar por los gobiernos centrales y locales, que tendría la potencial capacidad de poner fin al ciclo de la pobreza, eliminando la segregación entre barrios y aumentando el éxito educativo del alumnado más desfavorecido.

$\mathrm{Si}$ bien podrían considerarse ciertas limitaciones, como el tono excesivamente crítico de algunos de sus planteamientos (como su rechazo de manera generalizada a los constructos existentes, que puede dificultar el uso práctico de sus recomendaciones), o su escueto abordaje de ejemplos de análisis de datos eficaz (que podría haber sido un argumento efectivo a favor de los cambios metodológicos defendidos), lo cierto es que la obra ofrece reflexiones y sugerencias innovadoras para la superación de las desventajas educativas, a tener en cuenta tanto por responsables políticos como por académicos, retando a los investigadores a conservar su visión auto-crítica y depurar la construcción de sus metodologías de investigación.

Sandra Romero Martín, Universidad de Zaragoza sandraromero@unizar.es 